\title{
Interferometric survey of Be stars with the CHARA array
}

\author{
Yamina N. Touhami ${ }^{1}$, Douglas R. Gies ${ }^{1}$, Gail H. Schaefer ${ }^{2}$, Noel D. \\ Richardson $^{1}$, Stephen J. Williams ${ }^{1}$, Erika D. Grundstrom ${ }^{3}$, and M. \\ Virginia McSwain ${ }^{4}$ \\ ${ }^{1}$ Center for High Angular Resolution Astronomy, GSU, Atlanta, GA 30302, USA \\ ${ }^{2}$ The CHARA Array, Mount Wilson Observatory, Mount Wilson, CA 91023, USA \\ ${ }^{3}$ Physics and Astronomy Department, Vanderbilt University, Nashville, TN 37235, USA \\ ${ }^{4}$ Department of Physics, Lehigh University, Bethlehem, PA 18015, USA
}

\begin{abstract}
We present the first spatially resolved observations of circumstellar envelopes of 25 bright northern Be stars. The survey was performed with the CHARA Array interferometer in the K-band at intermediate and long baselines. The interferometric visibilities are well fitted by a viscous disk model where the gas density steeply decreases with the radius. Physical and geometrical parameters such as the density profile, the inclination, and the position angles of the circumstellar disks are determined. We find that the density radial exponent ranges between $n \approx 2.4-3.2$, which is consistent with previous IRAS measurements. We have also obtained simultaneous optical and near-IR spectrophotometric measurements, and found that the model reproduces well the observed disk IR-continuum excess emission. By combining the projected rotational velocity of the Be star with the disk inclination derived from interferometry, we give estimates of the equatorial rotational velocities of these Be stars.
\end{abstract}

Keywords. techniques: photometric, techniques: interferometric, stars: emission-line, Be

\section{Introduction and Observations}

Be stars are rapidly rotating B-type stars that manage to eject gas into a circumstellar disk (observed in $\mathrm{H}$ emission lines, an infrared flux excess, and linear polarization; Porter \& Rivinius. 2003). The IR flux excess emission results from bound-free and free-free emission, which increases in strength with wavelength (Dougherty et al. 1994). We have conducted the first interferometric Be star survey in the K-band using the CHARA Array. These observations cover a wide range of baselines from 30 to 331 meters. Because Be star disks are intrinsically variable on timescales of months to years, it was necessary to obtain contemporaneous spectrophotometry in order to model both the total flux and its angular distribution in the sky. We have obtained simultaneous near-IR spectroscopy of our sample using the NASA Infrared Telescope Facility and SpeX cross-dispersed spectrograph in 2006, and using the Mimir camera/spectrograph and Lowell Observatory Perkins Telescope in 2008 and 2009.

\section{Modeling the Visibility Measurments}

We calculate the theoretical visibility using a physical thick disk model that predicts given the near-IR emission the disk gas density (Gies et al. 2007) and compare it with visibilities for a simple uniform disk and Gaussian ellipsoid models. The disk is assumed to be axisymmetric and isothermal with a disk temperature equal to two thirds the stellar effective temperature. The model generates infrared images of the star surrounded by a 


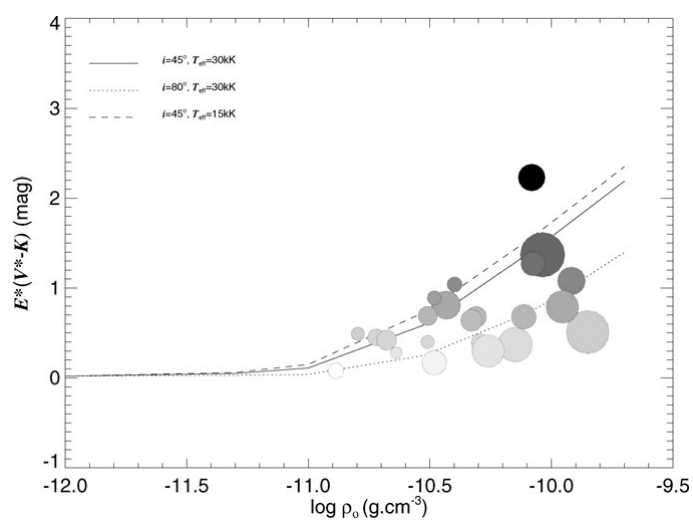

Figure 1. Theoretical IR-excess as a function of the disk base density for several model input parameters. The solid line is the default model with $i=45^{\circ}$ and $T_{\text {eff }}=30 \mathrm{kK}$. The dotted line represent the case of a higher inclination disk and the dashed line is for a medium inclination and cooler Be star.

circumstellar disk, computes the fast Fourier transform, predicts the visibility curve that is directly comparable to the observational data, and extracts the IR excess emission. Figure 1 shows a plot of the theoretical continuum IR excess as a function of the gas base density. The solid line is the color excess for a disk with an inclination of 45 degrees and an effective temperature of $30 \mathrm{kK}$. The dotted line is for 80 degrees inclination and $30 \mathrm{kK}$ effective temperature, the dashed line is for 45 degree inclination and $15 \mathrm{kK}$ effective temperature. We also plot in circles the interferometric results with circle sizes proportional to the obtained disk angular sizes.

\section{Discussion}

Our interferometric survey of nearby Be stars shows that all the stars display strong near-infrared emission that is coming from their circumstellar environments. The observations clearly indicate a resolved circumstellar disk around each star in our sample. We find that the thick disk model agrees well with the interferometric data, and that the best-fit values of the estimated gas densities are consistent with IRAS results. We also determine the angular size for each Be circumstellar disk. We find that the angularly resolved disks appear smaller in the lower opacity K-band continuum compared to that seen in the high opacity $\mathrm{H} \alpha$ line, which is consistent with the K-band excess forming mainly in the inner part of the disk (Touhami et al. 2010). The simultaneous spectroscopic survey shows that stars with strong $\mathrm{H} \alpha$ emission display an IR excess relative to the expected photospheric flux distribution. We find a good correlation between the size of the IR excess and the gas base density and the $\mathrm{H} \alpha$ equivalent-width. By combining the derived inclination witht the projected rotational velocity, we determine the equatorial rotational velocity of the central star. We find that Be stars are rapid rotators with equatorial velocities ranging between $0.7-0.9$ of their critical velocities.

\section{References}

Dougherty, S. M., Waters, L. B. F. M., Burki, G., Cote, J. et al. 1994, A\&JA, 290, 609

Gies, D. R., Bagnuolo, Jr., W. G., Baines, E. K., ten Brummelaar, T. A. et al. 2007, ApJ, 654, 527

Porter, J. M. \& Rivinius, T. 2003, PASP, 115, 1153

Touhami, Y., Richardson, N. D., Gies, D. R., Schaefer, G. H. et al. 2010, PASP, 122, 379 